\title{
Mobile Initiated Priority Access Solutions for Cellular Networks
}

\author{
Hsuan-Jung Su \\ Department of Electrical Engineering \\ National Taiwan University \\ Taipei, Taiwan \\ hjsu@cc.ee.ntu.edu.tw
}

\author{
On-Ching Yue \\ Department of Information Engineering \\ Chinese University of Hong Kong \\ Hong Kong \\ onching@ie.cuhk.edu.hk
}

\begin{abstract}
The rapid growth of the wireless communication networks has provided subscribers with economical and nearly ubiquitous wireless access throughout the world. As a result, not only the individual subscribers but also the subscribers in the government and public organizations have become dependent on the commercial wireless services to perform their duties. With this said, the commercial wireless service becomes a more costeffective means to assist national security and emergency preparedness as compared to the costly development of a private network. Sharing the commercial wireless services, however, could result in blocking of the security or emergency calls during or after a disaster when heavy traffic demand is placed on the surviving systems. In this paper, we discuss enhancements to the mobile stations that will make the user initiated priority access possible. These enhancements can operate with or without the priority access provisioned in the current or ongoing standards, and the additional complexity they impose is very marginal. With the combination of these enhancements, different levels of emergency call service can be provided to guarantee the success of emergency calls and shorten their setup time.
\end{abstract}

\section{MOTIVATION}

The rapid growth of the wireless communication networks has provided subscribers with economical and nearly ubiquitous wireless access throughout the world. As a result, not only the individual subscribers but also the subscribers in the government and public organizations have become dependent on the commercial wireless services to perform their duties. With this said, the commercial wireless service becomes a more cost-effective means to assist national security and emergency preparedness as compared to the costly development of a private network. Sharing the commercial wireless services, however, could result in blocking of the security or emergency calls during or after a disaster whien heavy traffic demand is placed on the surviving systems. It is therefore necessary to equip the commercial wireless service with a priority access mechanism to ensure the connection of a government emergency call. Priority access is also desirable in the third generation wireless networks which support mixed services with different quality of services.

In the existing commercial wireless network standards [7], or the standards in progress [4][5], priority access is provisioned. However, as will be detailed later, these priority access provisions are more for the purpose of traffic and overload control. The individual users may not be guaranteed to establish an emergency call when needed. Moreover, with the priority access functions not currently widely available, it is necessary to provide government officials or emergency workers with equipments that can always make emergency calls no matter the connecting network has priority access functions implemented or not. In this paper, we discuss enhancements to the mobile stations that will make the mobile initiated priority access possible. These enhancements can operate with or without the priority access provisioned in the current or ongoing standards, and the additional complexity they impose is very marginal. With the combination of these enhancements, different levels of emergency call service can be provided to guarantee the success of emergency calls and shorten their setup time. Under normal random access channel load, these performance enhancing techniques can reduce the mean acquisition time by up to $68 \%$. When the random access channel is overloaded, these techniques also improve substantially the probability of successful access attempts.

\section{BACKGROUND}

The current wireless networks separate the communication between a mobile station and a base station into two stages: access stage and traffic stage. When a mobile station enters a network, it listens to the pilot and synchronization channels broadcasted from the base station. Once the mobile station synchronizes with the network, it can extract from the base station's broadcast messages the information it needs to get access to the network. During the access stage, the mobile station transmits on the reverse link Random Access Channels $(\mathrm{RACH})$ trying to make the base station aware of $\mathrm{jts}$ existence. Since the base station does not have any information (e.g., timing, mobile identification, etc.) about the mobile station before the access stage, the access stage involves search and detection of the mobile access message. Once the base station successfully detects the access message, a traffic channel will be assigned to this mobile station and the regular call establishment process starts.

As the access and traffic stages are separated, they both impose a bottleneck on the network capacity. As a result, priority access can be performed on both stages. For the access stage under normal RACH load, the high priority mobile stations can increase their chance to be detected by the base station by using higher power or more frequent transmission. In the situation when the RACH is overloaded, individual priority enhancement may no longer work because the $\mathrm{RACH}$ is contention based and the heavy traffic on the RACH causes too high a mutual interference for any mobile station to be detected. A proper centralized priority access control is necessary in this situation. This so-called "overload control" is handled by the base station by assigning different transmission 
priorities to different mobile stations via the broadcast channel [4][7]. When the RACH is well functioning and the mobile - stations' origination messages can be detected by the base station, the base station can perform the traffic stage priority access control by assigning the traffic channels to the high priority users. This priority access control procedure is termed priority access service (PAS) [1] or priority access channel assignment (PACA) in cdma 2000 [1].

To summarize, individual access priority enhancements can increase the chance of a mobile station being detected when the RACH is not overloaded. These enhancements can operate with or without the base station priority access control. To avoid the overload situation and to enhance the access channel throughput, the base station overload control can be utilized. Once the access stage has passed and the mobile station enters the traffic stage, PACA can be used to give the emergency calls higher priority. This paper focuses on the individual access priority enhancements and provides some preliminary performance results. These enhancements and performance results are discussed in Section III. For completeness, base station overload control and priority access service are highlighted in Section IV and V, respectively.

\section{RANDOM ACCESS PRIORITY}

In this section we discuss the methods that a mobile station can implement individually to increase its access priority. These methods can be combined to provide different levels of enhancement in the access successfulness.

The reverse RACH consists of a number of time slots where the mobile stations can transmit their access preambles [3][5]. Upon the detection of a preamble, the base station can use the timing information extracted to decode the following access message [3], if there is any; or to inform the corresponding mobile station to send the access message to identify itself [5]. In a code division multiple access (CDMA) system, the preamble length can be longer than a slot. Due to low cross correlation between preamble sequences, a preamble can be detected as long as the preamble signal to interference (contributed by the preambles and traffic channels of other mobile stations) ratio (SIR) is high enough. Since the base station is not aware of the existence of the mobile station before a successful access, power control of the preamble is not possible. Hence the mobile station will start transmission with a predetermined power level. If the mobile station does not receive the acknowledgement (ACK) of its previous preamble transmission, it will retransmit the preamble with a higher power. Basically, the mobile station attempts blindly to get its preamble recognized by the base station. Without knowing the carrier phase of the signal from the mobile station, the base station detects the mobile preamble noncoherently. In addition, the arrival time of the mobile signal is not known to the base station due to variable distance between the mobile station and the base station, and the randomized delay the mobile station imposes on the preamble in order to avoid collision with the preambles of other mobile stations. As such, it is very important that the base station can successfully acquire a preamble and identify its timing before the end of the preamble. A probability of acquisition $P_{a c q}$ is defined to measure the performance of a RACH:

$$
P_{a c q}=\operatorname{Pr}\left(T_{a c q} \leq T_{h}\right)=\int_{0}^{T_{h}} f_{T_{a c q}}(\tau) d \tau
$$

where $T_{a c q}$ is the time spent by the base station to acquire a preamble, $T_{h}$ is the preamble length, and $f_{T_{a c q}}(\tau)$ is the probability density function (PDF) of the acquisition time.

The first method that can enhance the mobile station's probability of acquisition is to allow higher access transmission power. To evaluate the effect of transmission power, we assume a standard double dwell serial-parallel acquisition algorithm [6] at the base station. Precisely, there are $K$ double dwell serial search circuits operating in parallel, each searching a portion of the total timing uncertainty of $L$ chips. Assuming that each portion has $\omega$ chips $(L=K \omega)$, and each chip is sampled and divided into $l$ cells, each circuit will be testing $v=l \omega$ cells, among which at least $v-2 l$ can lead to false alarms. Within a search dwell, the fading is almost constant. The false alarm probability for noncoherent reception can be written as [6]

$$
P_{F i}=e^{-\Theta_{i} / V_{i}}, \quad i=1,2
$$

where $i$ is the dwell index and $\Theta_{i}$ is the corresponding threshold. $V_{i}=\gamma_{i} I_{0}$ is the noise plus interference energy, with $\gamma_{i}$ being the dwell (accumulation) time. The detection probability is given by

$$
P_{D i j}=\int_{\theta_{i} / V_{i}}^{\infty} e^{-\left(x+\mu_{i j}\right)} J_{0}\left(2 \sqrt{\mu_{i j} x}\right) d x, i=1,2 ; j=1 ; 2, \ldots, 2 l
$$

where $\mu_{i j}$ is the SIR of dwell $i$ at cell $j$ within one chip of the correct timing. The following branch transfer functions can be applied to the reduced acquisition state diagram [6], with $H_{0}(z)$ corresponding to the transfer function of the branches emerging from the $v-2 l$ nodes without the presence of the signal, and $H_{D}(z)$ and $H_{M}(z)$ being the detection and miss transfer functions, respectively, emerging from the superstate corresponding to the cells within one chip of the correct timing. $\gamma_{p}$ is the penalty for a false alarm at the second dwell.

$$
\begin{aligned}
& H_{0}(z)= \\
& z^{\gamma_{1}}\left(1-P_{F 1}\right)+z^{\gamma_{1}+\gamma_{2}} P_{F 1}\left(1-P_{F 2}\right)+z^{\gamma_{1}+\gamma_{2}+\gamma_{p}} P_{F 1} P_{F 2} \\
& H_{D}(z)=\sum_{j=1}^{2 l} P_{D 1 j} P_{D 2 j} z^{\gamma_{1}+\gamma_{2}} \prod_{j=1}^{j-1}\left[\left(1-P_{D I i}\right) z^{\gamma_{1}}\right]
\end{aligned}
$$




$$
\begin{aligned}
H_{M}(z) & =z^{2 / \gamma_{1}} \prod_{j=1}^{2 l}\left(1-P_{D 1 j}\right) \\
& +\sum_{j=1}^{2 l} P_{D I j}\left(1-P_{D 2 j}\right) z^{\gamma_{1}+\gamma_{2}} \prod_{i=1}^{j-i}\left[\left(1-P_{D 1 i}\right) z^{\gamma_{1}}\right]
\end{aligned}
$$

With these transfer functions, the PDF of the acquisition time, and hence the probability of acquisition, can be computed numerically. In Table 1, the probability of acquisition is shown using a cdma2000 RACH structure (namely, $L=512$ chips; an access probe containing a preamble of length $T_{h}$ which is two RACH frames $(40 \mathrm{~ms})$, and an access message of five frames (100ms) in length) [2][3], and the acquisition circuit parameter $K=6, l=2, \gamma_{1}=64$ chips, $\gamma_{2}=128$ chips, $\gamma_{p}=120 \mathrm{~ms}, P_{F 1}=0.01, P_{F 2}=0.05$.

Table 1: Probability of acquisition for different access preamble $E_{c} / I_{0}\left(E_{c}\right.$ : energy per chip)

\begin{tabular}{|c|c|}
\hline$E_{c} / I_{0}(\mathrm{~dB})$ & $P_{a c q}$ \\
\hline-9 & 0.95 \\
\hline-10 & 0.94 \\
\hline-11 & 0.92 \\
\hline-12 & 0.88 \\
\hline-15 & 0.48 \\
\hline-18 & 0.13 \\
\hline-21 & 0.04 \\
\hline
\end{tabular}

It is clear from Table 1 that the probability of acquisition increases with the transmission power. Therefore, if a mobile station is allowed to transmit with higher power, its probability of establishing an emergency call will be increased. Under normal RACH load (e.g., $E_{c} / I_{0}=-12$ to $-9 \mathrm{~dB}$ ) the probability of acquisition is usually high. When the RACH is overloaded and the SIR is very low (e.g., $E_{c} / I_{0}<-15 \mathrm{~dB}$ ), the probability of acquisition could drop substantially.

In the event where one access probe fails to be acquired, the transmission power can also be increased gradually for the subsequent retries to increase their acquisition probability. This adjustment of power for retransmissions is called "power ramping" [5]. If the mobile station does not receive the ACK after a maximum allowable number of access attempts or it receives a negative acknowledgement (NACK), it stops the access procedure and waits for a random amount of backoff time to restart the procedure.

The second priority access enhancement involves increasing the persistence of a mobile station, in other words, increasing the retransmission probability of access probes. For cdma2000, whenever an access probe fails, the mobile station will initiate another access probe with a random delay of 1-16 slots after the previous access probe. The duration of a slot equals to that of an access probe. Equivalently, the arrival rate of access probes is $1 / 16$ per slot. On top of this, a persistence factor can be assigned to alter the access probe arrival rate. For simplicity, assume that the power for every access probe is the same. Let the access probe arrival rate after the persistence adjustment be $G$. Except for the initial access probe, in every given slot, a successful acquisition requires two conditions: 1.) there is an access probe in that slot, and 2.) the access probe is acquired. Let $x$ be the mean acquisition time under the condition that the first access probe fails, then

and

$$
x-1=G P_{a c q}+\left(1-G P_{a c q}\right) x
$$

$$
x=1+\frac{1}{G P_{a c q}}
$$

The mean acquisition time $T_{A}$ can be computed as

$$
T_{A}=P_{a c q}+\left(1-P_{a c q}\right) x=1+\frac{1-P_{a c q}}{G P_{a c q}} \text { (slots) }
$$

Table 2: Mean acquisition time performance (in RACH slots) for different access persistence.

\begin{tabular}{|c|c|c|c|}
\hline $\begin{array}{c}E_{c} / I_{0} \\
(\mathrm{~dB})\end{array}$ & $\begin{array}{c}G \text { (access } \\
\text { probes/slot) }\end{array}$ & $\begin{array}{c}T_{A} \\
(\mathrm{sec})\end{array}$ & $P_{3 \mathrm{sec}}$ \\
\hline-9 & $1 / 16$ & 0.2579 & 1 \\
\hline-9 & $1 / 8$ & 0.1990 & 1 \\
\hline-12 & $1 / 16$ & 0.4455 & 1 \\
\hline-12 & $1 / 8$ & 0.2927 & 1 \\
\hline-15 & $1 / 16$ & 2.5667 & 1 \\
\hline-15 & $1 / 8$ & 1.3533 & 1 \\
\hline-18 & $1 / 16$ & 15.131 & 0.9454 \\
\hline-18 & $1 / 8$ & 7.6354 & 0.9471 \\
\hline-21 & $1 / 16$ & 53.900 & 0.5733 \\
\hline-21 & $1 / 8$ & 27.020 & 0.5771 \\
\hline
\end{tabular}

In Table 2, the performance for different $E_{c} / I_{0}$ (under normal or overload situations) and access probe arrival rates are shown in terms of mean acquisition time and the probability $\left(P_{3 \mathrm{sec}}\right)$ that an access attempt succeeds within 3 sec. Under normal RACH load $\left(E_{c} / I_{0}>-15 \mathrm{~dB}\right)$, an access attempt always succeeds in less than $3 \mathrm{sec}$. By allowing $3 \mathrm{~dB}$ higher transmission power and twice higher persistence (from $-12 \mathrm{~dB}, 1 / 16$ to $-9 \mathrm{~dB}, 1 / 8)$, the mean acquisition time can be reduced by more than $55 \%$. When the $\mathrm{RACH}$ is overloaded, similar arrangement can reduce the mean acquisition time by up to $91 \%$, and increase the probability of successful access attempt in $3 \mathrm{sec}$ by $36 \%$. These two enhancements do not incur any additional system complexity.

Increasing the access power and persistence alone may not be able to help mobile stations needing high priority, because the 
mobile stations have no means to know whether a $\mathrm{RACH}$ is overloaded with other mobile stations' access attempts. Thus, it helps if the high priority mobile stations are equipped with the capability to simultaneously transmit on different RACHs which may be on different carriers, or belong to the neighbor active base stations. In essence, higher access persistence provides the mobile station with more retransmissions and time diversity, while simultaneous transmissions on the RACHs of more than one carrier or more than one base station provide the mobile stations with frequency and spatial diversities, respectively, to prevent the mobile access from being delayed by an overloaded RACH.

To evaluate the enhancement of simultaneous RACH transmission, we consider the case where $N$ RACHs are available to the mobile station and the error events on these $\mathrm{RACHs}$ are independent. Let the acquisition probabilities on these RACHs be $P_{a c q}^{i}, i=1,2, \cdots, N$. As long as one of the simultaneous access probes is acquired, the access is considered successful. Thus, the equivalent acquisition probability is given by

$$
P_{a c q}=1-\prod_{i=1}^{N}\left(1-P_{a c q}^{i}\right)
$$

The enhancement provided by simultaneous access transmissions under normal RACH load is given in the upper part of Table 3. For fair comparison, the cases considered, except for the last row, have the same total power consumption. When more than one $\mathrm{RACH}$ is available, simultaneous access transmission can be implemented by either assigning the same persistence but lower power on the simultaneous RACHs, or assigning the same power but lower persistence on them. From Table 3 it can be seen that, under normal RACH load, the mean acquisition time can be reduced by $33 \%$ and $45 \%$, respectively, with these two implementations. Due to the sensitivity of $P_{a c q}$ to $E_{c} / I_{0}$, reducing too much the power on the simultaneous RACHs might hurt the performance. The results in Table 3 suggest that it is better to reduce the persistence rather than the power on the simultaneous RACHs. From the last row, it is also shown that with $3 \mathrm{~dB}$ higher power and simultaneous access transmissions, the mean acquisition time can be reduced by $68 \%$.

In the lower part of Table 3, it is assumed that all the RACHs are overloaded. With the same power consumption, again, simultaneous access transmissions reduce the mean acquisition time and increase the $P_{3 \mathrm{sec}}$. Consider the case where the interference on one $\mathrm{RACH}$ stays the same $\left(E_{c} / I_{0}=-21\right.$ $\mathrm{dB}$ ), while the interference on the other $\mathrm{RACH}$ is $3 \mathrm{~dB}$ lower. A mobile station having simultaneous access transmissions on both RACHs has $58 \%$ shorter mean acquisition time and $70 \%$ higher $P_{3 \mathrm{sec}}$, as compared to a mobile station trying to access through the worse $\mathrm{RACH}$.

Table 3: Mean acquisition time performance with fixed power.

\begin{tabular}{|c|c|c|c|c|}
\hline $\begin{array}{c}\text { Number } \\
\text { of } \\
\text { RACHs }\end{array}$ & $\begin{array}{c}E_{c} / I_{0} \\
(\mathrm{~dB}) \\
\text { per } \\
\mathrm{RACH}\end{array}$ & $\begin{array}{c}G \\
\text { (access } \\
\text { probes/ } \\
\text { slot/ } \\
\mathrm{RACH})\end{array}$ & $\begin{array}{c}T_{A} \\
(\mathrm{sec})\end{array}$ & $P_{3 \mathrm{sec}}$ \\
\hline 1 & -9 & $1 / 16$ & 0.2579 & 1 \\
\hline 2 & -12 & $1 / 16$ & 0.1727 & 1 \\
\hline 2 & -9 & $1 / 32$ & 0.1512 & 1 \\
\hline 4 & -15 & $1 / 16$ & 0.3167 & 1 \\
\hline 4 & -9 & $1 / 64$ & 0.1401 & 1 \\
\hline 1 & -12 & $1 / 16$ & 0.4455 & 1 \\
\hline $3 \mathrm{~dB}$ & & & \\
\hline \hline 1 & -21 & $1 / 16$ & 53.900 & 0.5733 \\
\hline 2 & -21 & $1 / 32$ & 52.803 & 0.8194 \\
\hline 4 & -21 & $1 / 64$ & 50.636 & 0.9674 \\
\hline 2 & -18, & $1 / 32$ & 22.844 & 0.9774 \\
& -21 & & & \\
\hline
\end{tabular}

In practice, providing frequency diversity requires the mobile stations to be able to monitor and transmit simultaneously on a wider range of frequency. Providing spatial diversity, on the other hand, is much easier, as the mobile station keeps monitoring the pilot signals of the neighbor base stations in the active set. In UMTS, access handoff [5], meaning the mobile station can switch to the RACH of a neighbor base station with a stronger pilot signal, is allowed. The additional requirement for simultaneous access through the RACHs of more than one base station is the capability to monitor the broadcast channels of more than one base station in order to see whether any of the previous simultaneous access probes have been acknowledged. Since the acknowledgement messages are usually transmitted at low data rate, the existing mobile stations should have enough processing power to handle such tasks.

\section{OVERLOAD CONTROL}

Due to the contention and mutual interfering nature of the $\mathrm{RACH}$, the throughput of the RACH will decline if it is overloaded. This is often the situation during or after a disaster when many users are trying to make calls through the surviving systems. In the worst case, all mobile stations will be using maximum power for their access preambles and the base station will still not be able to detect any of them. To make sure that some of the call requests can get through, the base station can classify the mobile stations and give each class an access persistence to control the RACH load. In both GSM [7] and cdma2000 [4], the mobile stations are divided into a maximum of 16 classes each assigned a different persistence. 
To avoid high interference in the overload situation, the base station can shut off some of the classes by giving them the lowest persistence. The overload control is not only useful for the RACH access control, it also makes sure that the throughput of the RACH is no larger than what the network traffic and/or broadcast channel (for ACK/NACK of the $\mathrm{RACH}$ ) can handle. Fairness and priority among the classes can be achieved by shuffling the persistence of the classes from time to time. As shown in Section III, the priority access enhancing methods proposed can operate with or without base station overload control.

\section{PRIORITY ACCESS SERVICE}

After the access stage, a call could still be blocked due to network congestion. When the network is congested, the priority access can be achieved by activating PAS or PACA. This method involves queuing the high priority access requests and setting up the call as soon as a traffic channel is available. Mechanisms similar to the existing wireline Government Emergency Telecommunications Service (GETS) can be used.

\section{CONCLUSION}

We have summarized the priority access in cellular networks in two stages: access stage and traffic stage. Some existing mechanisms were described and new methods and enhancements proposed. At the access stage, a mobile station can increase its priority individually by increasing its transmission persistence and/or power. These two enhancements do not incur any additional system complexity. In addition, simultaneous access transmissions on more than one $\mathrm{RACH}$ can also reduce the mean acquisition time. With the combination of these performance enhancing techniques, it was shown that, under normal RACH load, the mean acquisition time can be reduced by up to $68 \%$. These techniques also improve substantially the probability of successful access attempt within $3 \mathrm{sec}$, when the RACH is overloaded. On top of these mobile initiated priority access techniques, the base station can also perform overload control to make sure the RACH is functioning properly. At the traffic stage, priority access can be implemented by queuing the high priority calls and assign traffic channels to them as soon as the traffic channels are available.

\section{REFERENCES}

[1] C. Redding and C.-L. Taylor, "Priority Access Service in Cellular and PCS Networks," pp. 586-590, Vol. 1, Proc. IEEE Milcom 2001.

[2] 3GPP2 C.S0002, "Physical Layer Standard for cdma2000 Spread Spectrum Systems".

[3] 3GPP2 C.S0003, "Medium Access Control (MAC) Standard for cdma2000 Spread Spectrum Systems".

[4] 3GPP2 C.S0005, "Upper Layer (Layer 3) Signaling Standard for cdma2000 Spread Spectrum Systems".
[5] 3GPP TS 25.214 v3.4.0, "Physical Layer Procedures (FDD)".

[6] A. J. Viterbi, "CDMA: Principles of Spread Spectrum Communications", Addison-Wesley, Massachusetts, 1995.

[7] M. Mouly and M.-B. Pautet, "The GSM System for Mobile Communications," CELL \& SYS, 1992. 